\title{
Calculation of Strain Compensation Thickness for III-V Semiconductor Quantum Dot Superlattices
}

\author{
S. J. Polly ${ }^{\mathrm{a}}$, C. G. Bailey ${ }^{\mathrm{b}}$, A. J. Grede ${ }^{\mathrm{c}}$, D. V. Forbes ${ }^{\mathrm{a}}$, S. M. Hubbard ${ }^{\mathrm{a}}$ \\ ${ }^{a}$ Rochester Institute of Technology, Rochester, NY 14623, USA \\ ${ }^{b}$ Old Dominion University, Norfolk, VA 23529, USA \\ ${ }^{c}$ The Pennsylvania State University, University Park, PA, 16802, USA
}

\begin{abstract}
Models based on continuum elasticity theory are discussed to calculate the necessary thickness of a strain compensation (SC) layer for a superlattice (SL) of strained quantum wells (QW) or quantum dots (QD). These models are then expanded to cover material systems (substrates, QW or QD, and SC) composed of AlP, AlAs, AlSb, GaP, GaAs, GaSb, InP, InAs, or InSb, as well as the ternary, quaternary, and higher order material alloys possible in the $\mathrm{Al} / \mathrm{Ga} / \mathrm{In} / \mathrm{P} / \mathrm{As} / \mathrm{Sb}$ systems. SC thickness calculation methods were compared against dynamical scattering simulations and experimental $\mathrm{X}$-ray diffraction measurements of the InAs/GaP/GaAs QD/SC/Substrate superlattices of varying SC thickness. Based on the reduced (but not eliminated) strain present, a further modified strain compensation thickness is calculated to maximize the number of SL repeat units before the onset of misfit dislocations is also calculated. These models have been assembled into a free application on nanoHUB for use by the community.
\end{abstract}

Keywords: B2. Semiconducting III-V materials; A3. Quantum wells; A3. Quantum dots; A1. Low dimensional structures; A1. High resolution X-ray diffraction; A1. Strain compensation

\section{Introduction}

Quantum dot (QD) superlattices (SL) have become an important part of optoelectronic device research and development, with applications in tunable laser diodes [1] and low energy photodetectors [2]. They have been of particular interest to the photovoltaics community, where they are used for bandgap engineering [3], and in efforts to create an intermediate band solar cell [4, 5, 6].

Epitaxial QD growth, performed in situ using metalorganic vapor phase epitaxy (MOVPE), or molecular beam epitaxy (MBE), relies on a lattice mismatch between the QD material and the substrate, inducing the Stranski-Krastanov growth mode. As multiple layers of QDs are grown, the overall strain in the material increases, leading to formation of misfit and threading dislocations which propagate along the growth direction as additional layers are deposited. These defects then cause an increase in minority carrier recombination which degrades the open-circuit voltage and power conversion efficiency [7]. While the local strain around the QDs is necessarily inhomogeneous [8], the incorporation of a uniform strain compensation (SC) layer, with opposite strain from the QD to the substrate, can balance the global strain of the overall superlattice thus maintaining nominal optoelectronic device performance [9, 10].

Email addresses: sjpvpr@rit.edu (S. J. Polly), cgbailey@odu.edu (C. G. Bailey), ajg324@psu.edu (A. J. Grede), smhsps@rit.edu (S. M. Hubbard)

Preprint submitted to Elsevier

August 4, 2016

(C) 2016. This manuscript version is made available under the Elsevier user license http://www.elsevier.com/open-access/userlicense/1.0/ 
Determining the optimal thickness of a SC layer relies on knowledge of the size, shape, and areal density of the QDs, as well as properties of the substrate, QD, and SC material systems. While the GaAs / InAs QD / GaP SC system has been extensively studied, moving to alternative combinations of material systems, such as InGaAs/InGaP [11] or others, requires information about the elastic stiffness constants of the new materials, which may not be available experimentally.

This work uses and expands upon previously established methods to determine the nominal thickness of a superlattice SC layer using continuum elasticity theory (CET). Three methods are compared: compensation of quantum wells using CET alone [12], a modified CET method to include QDs, treating them as cylinders with detached wetting layers [13], and introduces a second modified CET method which treats the QDs as oblate-hemispheroids with attached wetting layers. The methods for balancing 3D structures with a $2 \mathrm{D}$ SC layer result in a global strain balance condition, however some areas are necessarily over-compensated while the rest of the film is under-compensated. A method of determining the critical number of repeat units before the onset of misfit dislocations based on these two methods is introduced and discussed. The critical SL thickness is then used to further refine the calculated SC thickness to maximize the number of SL layers before the onset of misfit dislocations.

To determine the necessary SC thickness for an arbitrary combination of (001) oriented cubic material systems, a calculator was developed using MATLAB/GNU Octave. The output can be used as an educated starting point to a systematic evaluation of optimal SC thickness for a given set of material systems and QD parameters. This program calculates the required SC thickness for QDs and their associated wetting layer (WL). It can also be used to calculate SC thickness for QWs, or to determine QD volume and effective QD material coverage based on QD properties. From these, an estimate of the critical, and maximum, number of SL repeat units before the onset of misfit dislocations is also presented.

\section{Theory}

\subsection{Continuum Elasticity Theory - $Q W$}

A method, described presently, to determine the thickness necessary to compensate the strain of a cubic (001)-oriented pseudomorphically grown quantum well with an oppositely strained SC layer by minimizing the average strain energy density was developed by Ekins-Daukes, Kawaguchi, and Zhang [12] using continuum elasticity theory (CET). Beginning with the pseudomorphic assumption, strain in $x$ and $y$ (in-plane) in the QW must be equal, shear strain is zero, and strain in the growth direction $z$ (out-of-plane) is a function of the strain in $x$ through the biaxial Poisson ratio. For a highly symmetric cubic lattice, these conditions result in the following stress $(\sigma)$-strain $(\epsilon)$ relationship

$$
\left(\begin{array}{c}
\sigma_{x x} \\
\sigma_{y y} \\
\sigma_{z z} \\
\sigma_{y z} \\
\sigma_{z x} \\
\sigma_{x y}
\end{array}\right)=\left(\begin{array}{cccccc}
C_{11} & C_{12} & C_{12} & 0 & 0 & 0 \\
C_{12} & C_{11} & C_{12} & 0 & 0 & 0 \\
C_{12} & C_{12} & C_{11} & 0 & 0 & 0 \\
0 & 0 & 0 & C_{44} & 0 & 0 \\
0 & 0 & 0 & 0 & C_{44} & 0 \\
0 & 0 & 0 & 0 & 0 & C_{44}
\end{array}\right)\left(\begin{array}{c}
\epsilon_{x x} \\
\epsilon_{x x} \\
\frac{-2 C_{11}}{C_{11}} \epsilon_{x x} \\
0 \\
0 \\
0
\end{array}\right)
$$

using the elastic stiffness constants for the $\mathrm{QW}$ material $C_{11}, C_{12}$, and $C_{44}$. This simplifies to

$$
\sigma_{x x}=\sigma_{y y}=\left(C_{11}+C_{12}-\frac{2 C_{12}^{2}}{C_{11}}\right) \epsilon_{x x}
$$


with all additional components of stress equal to zero. Strain in the QW is defined

$$
\epsilon_{1}=\frac{a_{0}-a_{1}}{a_{1}}
$$

using the unstrained lattice constant of the substrate, $a_{0}$, and QW, $a_{1}$. It is useful at this point to define the biaxial modulus for a particular layer $l$ as

$$
A_{l}=C_{11, l}+C_{12, l}-\frac{2 C_{12, l}^{2}}{C_{11, l}}
$$

from Equation 2

From here, the average strain energy density in a two-layer system composed of a strained QW with thickness $t_{1}$ and an oppositely strained SC with thickness $t_{2}$ is

$$
U_{a v}=\frac{U_{1} t_{1}+U_{2} t_{2}}{t_{1}+t_{2}}
$$

with the strain energy density of a layer $l$ given by

$$
U_{l}=\frac{1}{2}\left(\sigma_{x x, l} \epsilon_{x x, l}+\sigma_{y y, l} \epsilon_{y y, l}+\sigma_{z z, l} \epsilon_{z z, l}\right)=A_{l} \epsilon_{l}^{2}
$$

and simplified with the use of Equations 2 and 4 The strain of both strained layers can be defined in terms of the first

$$
\epsilon_{i}=\frac{a_{1}}{a_{i}} \epsilon_{1}+\frac{a_{1}-a_{i}}{a_{i}}
$$

leaving $U_{a v}$ as a function with one variable, $\epsilon_{1}$. The strain balanced condition for the QW/SC system is then found by setting the derivative of $U_{a v}$ with respect to $\epsilon_{1}$, or average in-plane stress, to zero

$$
\frac{\partial U_{a v}}{\partial \epsilon_{1}}=\frac{2}{t_{1}+t_{2}}\left(t_{1} A_{1} \epsilon_{1}+t_{2} A_{2} \epsilon_{2} \frac{a_{1}}{a_{2}}\right)=0 .
$$

This can be solved using known thicknesses to determine the necessary substrate lattice constant for a strain balanced bilayer. The rearrangement of Equation 8 to determine the necessary thickness of a strain compensation layer to match a strained superlattice to the substrate was explicitly presented by Bailey et al. [13], shown here using subscripts consistent with this manuscript

$$
t_{S C}=t_{Q W} \frac{A_{Q W} a_{S C}^{2}\left(a_{\mathrm{Sub}}-a_{Q W}\right)}{A_{S C} a_{Q W}^{2}\left(a_{S C}-a_{\mathrm{Sub}}\right)} .
$$

where $S C$ is the strain compensation layer, $Q W$ is the strained quantum well layer, and Sub is the substrate. A diagram of this structure is shown in Figure 1 a.

The work presented by Ekins-Daukes et al. [12] can be presented in a more general form to include an arbitrary number $n$ of strained layers (relative to the substrate) by using the average elastic energy density

$$
U_{a v}=\frac{\sum_{i=1}^{n} A_{i} \epsilon_{i}^{2} t_{i}}{\sum_{i=1}^{n} t_{i}}
$$


and the strain defined in Equations 3 and 7

Minimizing the average elastic energy density by solving for a zero average in-plane stress yields the required substrate lattice constant for any given set of materials

$$
a_{0}=\frac{\sum_{i=1}^{n}\left(A_{i} t_{i} a_{i} \prod_{\substack{k=1 \\ k \neq i}}^{n} a_{k}^{2}\right)}{\sum_{i=1}^{n}\left(A_{i} t_{i} \prod_{\substack{k=1 \\ k \neq i}}^{n} a_{k}^{2}\right)}
$$

or in terms of Equation 9, the required final SC thickness assuming all other layer thicknesses are fixed

$$
t_{S C}=t_{n}=\frac{-a_{n}^{2} \sum_{i=1}^{n-1}\left(-A_{i} t_{i} a_{i} \prod_{\substack{k=1 \\ k \neq i}}^{n-1} a_{k}^{2}+A_{i} t_{i} a_{0} \prod_{\substack{k=1 \\ k \neq i}}^{n-1} a_{k}^{2}\right)}{A_{n}\left(a_{0}-a_{n}\right) \prod_{i=1}^{n-1} a_{i}^{2}} .
$$

Here, the layer is denoted by the iterator subscript number, illustrated in Figure 1p, where 0 is the substrate, 1 is the QW material, $n$ is the SC material, and the other iterators are arbitrary. This calculates a strain compensation layer thickness to null the sum of the strains of all included layers, while taking into account their stiffness parameters. These equations include the explicit assumptions that $a_{1} \neq a_{0} \neq a_{n}$, and that $a_{n}$ produce strain of opposite sign to the effective lattice constant of layers $i=1$ through $i=(n-1)$. The values of any other $a_{i}$ may be equal to $a_{0}$, however this completely removes the effect of that particular layer from the model. For example, if $n=3$ and $a_{2}=a_{0}$, Equation 12 simplifies to Equation 9 . Because the component of the stress tensor in the growth direction is zero under the pseudomorphic assumption, the addition of buffer layers lattice-matched to the substrate, as are often included in strained superlattices, can not be taken into account as stress-relief using this method. The remainder of this manuscript assumes only two layers are involved in strain compensation: the QD or QW and the SC. In the case of QDs however, some non-contributing inter-layer is assumed to planarize the QDs subsequent to additional layer growth for simplification of geometric factors.

\subsection{Modified Continuum Elasticity Theory - $Q D$ as Cylinder}

Bailey et al. went on to modify the CET approach to include 3D quantum dots by introducing a weighting factor based on QD areal density $\rho$, height $h$, and diameter $d$, to compensate both the fractional area of the film made of QDs, and the remaining area, which is only the QD wetting layer (WL) [13]. A diagram of this structure is shown in Figure 15. This method treats the QD as a cylinder, with a volume

$$
V_{Q D, \text { Cyl. }}=\frac{\pi d^{2} h}{4}
$$

Two strain compensation layer thicknesses are calculated, based on Equations 9 and 4 the first to compensate just the WL thickness, $t_{W L}$, denoted $t_{S C, W L}$ using $t_{Q W}=t_{W L}$, and the second to compensate the QD, $t_{S C, Q D, C y l}$, using $t_{Q W}=h$. These thicknesses are weighted by the fractional areal density of QDs giving an appropriate SC thickness for the superlattice (SL)

$$
t_{S C, S L, \text { Cyl. }}=\rho \sigma t_{S C, Q D, \text { Cyl. }}+(1-\rho \sigma) t_{S C, W L}
$$


using the product of $\rho$, the 2D density of QDs, and the QD base area

$$
\sigma=\frac{\pi d^{2}}{4} .
$$

Here, the WL is a QW with cylinder-voids, filled with QDs, based on the density of QDs. This treats the QD and WL as non-overlapping entities. This method may also be appropriate for QDs with no wetting layer [14].

\subsection{Modified Continuum Elasticity Theory - QD as Oblate-Hemispheroid}

This work introduces an alternate weighted CET method of determining effective QD thickness: treating the $\mathrm{QD}$ as half of an oblate spheroid, with a volume

$$
V_{Q D, \text { Obl. }}=\frac{\pi d^{2} h}{6} .
$$

As with the previous method using Equations 9 and 4 , one strain compensation layer thickness is calculated for the WL $t_{S C, W L}$, using $t_{Q W}=t_{W L}$, and the second to compensate the quantum dot $\left(t_{S C, Q D, C y l}\right)$ using an effective $\mathrm{QD}$ thickness for $t_{Q W}$ of $t_{Q D, \text { Obl. }}=\rho V_{Q D, \text { Obl. }}$ which averages the volume of a $\mathrm{QD}$ against the QD density. This leads to a weighted SC thickness

$$
t_{S C, S L, \mathrm{Obl} .}=t_{S C, Q D, \mathrm{Obl} .}+t_{S C, W L}
$$

as the sum of the two components. This treats the QD and the WL as separate and overlapping entities, which may more appropriately reflect the geometry and hight information taken from high resolution imaging techniques such as atomic force microscopy (AFM) or transmission electron microscopy (TEM). A diagram of this structure is shown in Figure $1 \mathrm{~d}$.

\section{Material Parameters}

Several material parameters must be known to perform these calculations for a given system. Some of these parameters are well studied and readily available in the literature, such as lattice constant, while others may not be directly known, such as elastic stiffness constants for ternary, quaternary, and higher order material systems.

\subsection{Lattice Constants}

Lattice constants of binary compounds were taken from Vurgaftman, Meyer, \& Ram-Mohan [15]. Values for other alloys were determined by a weighted average of their binary constituents for evaluation of ternary, quaternary, and higher order compound lattice constants of arbitrary composition. In the case of ternary compounds, this weighting method simplifies to Vegard's law [16].

\subsection{Elastic Stiffness Constants}

Stiffness constants of binary compounds were also taken from Vurgaftman et al. [15]. Those of ternary, quaternary, and higher order compounds were calculated in two ways: interpolation (i) using the weighted average method described for lattice constants (as suggested by Vurgaftman et al.), or through an empirical (e) relationship

$$
\ln \left(C_{j k}\right)=\alpha_{j k} \ln (a)+\beta_{j k}
$$


presented by Adachi relating $C_{11}$ and $C_{12}$ to the lattice constant by a fit against measured data of binary compounds [17, pp. 47-49]. Here $C$ is the stiffness constant, while $j$ and $k$ are the indices of the stiffness coefficient. $\alpha$ and $\beta$ are coefficients extracted from a least-squares regression against experimental data.

The $C_{11}$ and $C_{12}$ fit Adachi uses includes values of materials (such as $\mathrm{BN}$ ) with significantly smaller lattice constants than those within the scope of this paper. Here, $C_{11}$ and $C_{12}$ values from [15] were re-fit only for compounds with $\mathrm{Al}, \mathrm{Ga}$, and In group III elements with $\mathrm{P}, \mathrm{As}$, and $\mathrm{Sb}$ group $\mathrm{V}$ elements. This reduced the $R^{2}$ error in matching the smaller subset of material systems used here; for materials outside the scope of this paper, Adachi's coefficients are more appropriate. A comparison of these parameters are shown in Figures $2\left(C_{11}\right)$ and $3\left(C_{12}\right)$. The coefficients determined for this work are shown in Table 1 coefficients from Adachi can be found in reference [17].

\subsection{Model Example}

With these components assembled, the required strain compensation for a wide range of material systems can be calculated. An example is shown in Figure 4 , where the required thicknesses of a SC layer composed of $\operatorname{GaAs}_{(y)} \mathrm{P}_{(1-y)}$ was calculated for InAs QDs grown on GaAs with a density of $5 \times 10^{10} \mathrm{~cm}^{-2}$, height of $2 \mathrm{~nm}$, and diameter of $25 \mathrm{~nm}$, which are typical for this system [13, 18]. For the CET model, the QW is assumed to be the same height as the QD, $2 \mathrm{~nm}$. While it is unlikely that a layer over 6 monloayers (ML) thick of InAs could grow coherently without relaxation on GaAs, this value was chosen to compare the three methods using a known QD height of $2 \mathrm{~nm}$. Using pure GaP as the SC material, both MCET models produce similar results between 2.0 and 2.6 ML, while the CET SC thickness is larger at 6.4 to 7.3 ML-this is expected as the effective coverage of InAs is lower for the QD system (2 nm thick InAs QDs in some places based on their density) than the QW system ( $2 \mathrm{~nm}$ thick InAs QW everywhere). Differences between the MCET models become more apparent as the concentration of arsenic, $y$, increases. For example, at $y=0.85$, the MCET results range from 16 to $22 \mathrm{ML}$. As the SC composition approaches GaAs, the necessary thickness approaches infinity.

The models presented here were compared against experimental results from five superlattice structures grown by MOVPE incorporating 10x layers of InAs quantum dots and GaP-based SC, with thicknesses of 2.8, 3.5, 4.2, 5.0, and 5.7 ML, using methods discussed elsewhere [13, 9, 18].

The samples were measured using $\omega-2 \theta$ high resolution X-ray diffraction (HRXRD) about the (004) reflection revealing superlattice satellite peaks. The position of the $0^{\text {th }}$ order $\theta_{S L}$ peak was often obscured under the intensity of the main Bragg peak, and was determined by fitting a Gaussian function to each of the two $\pm 1^{s t}$ order peaks and averaging their position to determine the relative offset of the $0^{\text {th }}$ order peak from the Bragg peak. Based on the quality of SL peaks, Pendellösung fringes, and previous TEM images of similar samples [13], these films appeared defect-free and fully strained. While the $0^{\text {th }}$ order peak location can be used to extract out-of-plane strain, a conclusion of Ekins-Daukes et al. was that this value may not be useful to assess in-plane strain balancing [12]. Therefore, to test the efficacy of the various models presented here, the experimental results were compared against simulation. Dynamical X-ray scattering calculations were performed using LEPTOS by Bruker. The simulated structures used the effective InAs thickness as calculated by the models, and the SC thicknesses from the experimental samples. The location of the simulated $0^{\text {th }}$ order $\theta_{S L}$ (again, relative to the Bragg peak) was determined and compared with the experimental result creating a Delta SL0 Peak.

These results are presented compared against the model in Figure 5. Here, the x-axis is the required SC thickness predicted by each model subtracted from the experimentally grown thicknesses. The y-axis is the calculated location of the $0^{\text {th }}$ order SL peak subtracted from the experimentally determined $0^{\text {th }}$ order SL peak location (Delta Thickness), both relative to the Bragg peak to normalize any experimental offset. Linear fits, with extrapolation to extend past $\mathrm{x}$ - and $\mathrm{y}$-intercepts, are also plotted. 
The particular model best suited for the material system would exhibit a $0^{\text {th }}$ order SL peak experimentally where predicted by the dynamical scattering calculation (producing no delta in peak location, therefore along the $y$-axis), occurring when the grown thickness matches what was prescribed by the model (producing no delta in thickness, therefore along the x-axis); ideally, the best model would produce a nominally strain balanced system resulting in a point at the origin of Figure 5. While all of the grown thickness of $\mathrm{SC} \mathrm{GaP} \mathrm{from} \mathrm{this} \mathrm{set} \mathrm{of} \mathrm{experiments} \mathrm{were} \mathrm{too} \mathrm{thin} \mathrm{(undercompensated,} \mathrm{in} \mathrm{terms} \mathrm{of} \mathrm{the} \mathrm{CET} \mathrm{models)} \mathrm{or}$ too thick (overcompensated, in terms of the MCET models), the extrapolation of the MCET using Oblate Hemispheroids directly crosses the origin, allowing for experimental validation of it to predict the required $\mathrm{SC}$ thickness for this particular material system combination.

\subsection{Critical QD Superlattice Thickness}

The critical thickness, beyond which misfit dislocations begin to form, of a strained layer superlattice has been shown previously by Matthews and Blakeslee [19]. It is important to note that this method does not take into account the kinetics of dislocations during growth, which may allow thicker layer growth before dislocation formation, however, attempting to integrate kinetics requires estimations of additional unknown energies for the broad range of materials systems discussed, so only the equilibrium method is used to predict the minimum number of repeat layers possible to grow before the possibility of plastic deformation [20]. In this work, nominally, the strain has been properly balanced using one of the above methods, and an infinite number of repeat units can be grown without accumulation of strain; indeed, this is true for 2D QW calculations using the CET from Section 2.1. However, the MCET methods rely on weighting the strain compensation thickness based on the density and thickness of the component QDs and WL, resulting in a SC matched to the overall system, but necessarily under-compensating the strain caused by the QD, and over-compensating the WL. A critical thickness of the superlattice can be calculated using the transcendental Matthews-Blakeslee formula

$$
h_{c, l}=\frac{b_{l}}{2 \pi \epsilon_{0, l}} \frac{1-v \cos ^{2}(\gamma)}{(1+v) \cos (\lambda)}\left(\ln \left(\frac{h_{c, l}}{b_{l}}\right)+1\right)
$$

for each of two cases $l$ : a multilayer comprised of only QD/SC (the under-compensated portion), and a multilayer composed of only WL/SC (over-compensated).

Equation 19 assumes a (1 110$)$ Burgers vector magnitude of

$$
b_{l}=\frac{a_{S L, l}}{2} \sqrt{1^{2}+1^{2}+0^{2}}=\frac{a_{S L, l}}{\sqrt{2}}
$$

which requires an effective lattice constant for the superlattice

$$
a_{S L, l}=\frac{A_{1} t_{1} a_{1} a_{2}^{2}+A_{2} t_{2} a_{2} a_{1}^{2}}{A_{1} t_{1} a_{2}^{2}+A_{2} t_{2} a_{1}^{2}}
$$

from the solution to Equation 8][12], where subscripts 1 and 2 denote the QD and SC films, respectively.

Strain is defined as

$$
\epsilon_{0, l}=\frac{\left|a_{S L, l}-a_{\mathrm{Sub}}\right|}{a_{\mathrm{Sub}}}
$$

and the (l0 0 1) Poisson ratio of

$$
v=\frac{C_{12}}{C_{11}+C_{12}}
$$


was chosen as whichever material (SC or QD) has the larger value, to predict a worst-case result and underestimate the critical number of SL repeat units.

Angles $\gamma$ and $\lambda$, both assumed as $60^{\circ}$ for this system, are defined as the angle between the dislocation line and Burgers vector, and the angle between the slip direction and the direction in the slip plane perpendicular to the intersection line of the slip plane and interface, respectively. These values, as well as those for the Burgers vector, were taken from the literature as typical for these III-V materials [19, 21].

The tetragonally distorted lattice constants of the strained layers

$$
a_{\perp}=\left(a_{\text {layer }}-a_{\text {Sub }}\right)\left(1+\frac{2 C_{12, \text { layer }}}{C_{11 \text {,layer }}}\right)+a_{\text {Sub }}
$$

can be calculated to ease presenting values in terms of monolayers or to corroborate against microscopy [22].

\subsection{Maximum Superlattice Method}

Once the critical thickness for the QD/SC and WL/SC pairs are calculated, the integer number of repeat units to the superlattice that can be grown before the critical thickness is reached

$$
\chi_{l}=\left\lfloor\frac{h_{c, l}}{t_{S C, S L}+t_{Q}}\right\rfloor
$$

can be calculated for each, where $t_{Q}$ is defined as the larger of QD height $h$, or $t_{W L}$, for the Cylinder method, and as $h+t_{W L}$ for the Oblate Hemispheroid method. A final integer value is reported using the floor function for consistency in showing only complete SL repeat units. Results of $\chi$ using the parameters from Section 3.3 are shown in Figure 6. These calculations are necessarily underestimates, as in the case of the QD/SC system there was not actually a layer as thick as a QD across the whole of the sample, but it gives a confident minimum to the number of repeat units that can be grown before misfit dislocations begin to form. In the case of this system, the QDs are the limiting factor to the number of repeat units and will begin to cause defects due to compressive plastic deformation from under-compensation with the SC (black lines) before the over-compensated WL critical limits are exceeded (red lines). Additionally, as the composition of the SC approaches GaAs, the total number of repeat units increases. Increased As composition causes increased SC thickness, meaning the SC makes up a larger percentage of the total SL repeat unit and the effective lattice constant approaches the GaAs substrate. This reduces the effective strain for both QD/SC and WL/SC systems, enabling a larger critical thickness and as a result, a larger number of repeat layers.

The final calculated SC thickness, for either of the weighted QD methods, may be further refined with the goal of maximizing the number of superlattice repeat units. An iterative method

$$
\begin{gathered}
\chi_{\max }=\chi_{l}^{n}=\frac{h_{c, l}^{n}}{m_{n} \times t_{S C, S L}+t_{Q}} \\
h_{c, l}^{n}=h_{c, l}\left(\epsilon_{0, l}=\epsilon_{0, l}^{n}\right) \\
\epsilon_{0, l}^{n}=\epsilon_{0, l}\left(a_{S L, l}=a_{S L, l}^{n}\right) \\
a_{S L, l}^{n}=a_{S L, l}\left(t_{2}=m_{n} \times t_{S C}\right) \\
m_{n}=\left\{\begin{array}{lll}
m_{n-1} & \chi_{Q D}^{n-1} \approx \chi_{W L}^{n-1} & n \neq 0 \\
m_{n-1}+\frac{1}{2^{n-1}} & \chi_{Q D}^{n-1}<\chi_{W L}^{n-1} & n \neq 0 \\
m_{n-1}-\frac{1}{2^{n-1}} & \chi_{Q D}^{n-1}>\chi_{W L}^{n-1} & n \neq 0 \\
r & n=0
\end{array}\right.
\end{gathered}
$$


was used to determine the SC thickness that produced the largest critical SL thickness for each of two cases $l$ simultaneously: the QD/SC system, and the WL/SC system. An initial SC thickness $t_{S C, S L}$ was calculated using the methods described previously, which was then increased or decreased by multiplication with $m$ through $n$ iterations (indexed at 0 ) until $\chi_{Q D}^{n} \approx \chi_{W L}^{n}$ to some finite precision. Initially, if $\chi_{Q D}^{0}<\chi_{W L}^{0}, m$ is set to the smallest integer value $r$ which causes $\chi_{Q D}^{0}>\chi_{W L}^{0}$; otherwise $m=1$. The iterative process follows where the iterator $n$ is used primarily as a label, it is only meant as an exponent where $2^{n-1}$. Using this method recursively, the multiplier $m$ approaches a value between $r \pm 1$, allowing $m_{n} \times t_{S C}$ (the new $\mathrm{SC}$ thickness) to cover the entire possible range between $t_{S C, Q D}$ and $t_{S C, W L}$. This method remains suitable even for situations where the height of the QD is less than thickness of the WL. Again, a final integer value of $\left\lfloor\chi_{\text {max }}\right\rfloor$ is reported. In the case of this example system, the SC thickness to maximize the superlattice is thicker than the initially calculated value shown in Figure 4 The over-compensation of the WL increased and the under-compensation of the QD decreased, leading to an overall increase in the limiting number of superlattice repeat layers shown in Figure 6 (green lines). The resulting SC thickness lies between (though not necessarily at the mean) of the QD/SC and WL/SC components, enabling a minimum estimate of the maximum SL possible to grow in the given system.

\section{Software}

An application incorporating the various functions described in this manuscript was developed in MATLAB/GNU Octave and is freely available for use on nanoHUB [23]. User inputs include the material composition of the substrate, QD or QW, and strain compensation layer, as well as the density, height, diameter, and WL thickness for the QDs. Outputs include the calculated lattice constants and elastic stiffness constants for all materials involved, as well as the required strain compensation layer thickness for the system and the effective coverage of QD material using all of the calculation methods described here. This software calculates parameters for substrates, QD or (QW), and SCs made of AlP, AlAs, AlSb, GaP, GaAs, GaSb, InP, InAs, InSb, as well as the ternaries, quaternaries, and other higher order alloys possible between those binary systems.

\section{Conclusion}

Three models to calculate the strain compensation layer thickness necessary to offset a strained layer composed of QDs were presented, and the steps required to extend their application across a wide range of binary, ternary, quaternary, and higher order material systems was discussed. Using these methods, the maximum number of repeat units for a SL was also calculated before the onset of misfit dislocations. The best model to use for a particular application depends strongly on the specific geometries involved, however the results presented by the various models provide an educated starting point to begin in an otherwise unknown system.

\section{Acknowledgment}

This work was supported by the National Science Foundation: DMR-0955752; US Department of Energy: DE-FG36-08GO18012; and the US Department of Education Graduate Assistance in Areas of National Need (GAANN) Fellowship: P200A090225. Materials growth for this work was supported through NASA: SAA3-844. 


\section{References}

[1] K. A. Fedorova, M. A. Cataluna, I. Krestnikov, D. Livshits, E. U. Rafailov, Broadly tunable highpower InAs/GaAs quantum-dot external cavity diode lasers Optics Express 18 (18) (2010) 19438. doi:10.1364/0E.18.019438.

URL http://www.opticsinfobase.org/oe/fulltext.cfm?uri=oe-18-18-19438\&id= 205412

[2] A. Barve, S. Lee, S. Noh, S. Krishna, Review of current progress in quantum dot infrared photodetectors, Laser \& Photonics Reviews 4 (6) (2010) 738-750. doi : 10. 1002/lpor . 200900031. URL http://onlinelibrary.wiley.com/doi/10.1002/lpor.200900031/abstract

[3] S. M. Hubbard, C. Bailey, S. Polly, C. Cress, J. Andersen, D. Forbes, R. Raffaelle, Nanostructured photovoltaics for space power. Journal of Nanophotonics 3 (1) (2009) 031880. doi : doi : 10.1117/ 1.3266502. URL http://spiedigitallibrary.org/jnp/resource/1/jnoacq/v3/i1/p031880_s1

[4] A. Luque, P. G. Linares, E. Antoln, I. Ramiro, C. D. Farmer, E. Hernndez, I. Tobas, C. R. Stanley, A. Mart, Understanding the operation of quantum dot intermediate band solar cells, Journal of Applied Physics 111 (4) (2012) 044502-044502-12. doi : doi : 10.1063/1.3684968. URL http://jap.aip.org/resource/1/japiau/v111/i4/p044502_s1

[5] Y. Shoji, K. Akimoto, Y. Okada, Self-organized InGaAs/GaAs quantum dot arrays for use in highefficiency intermediate-band solar cells, Journal of Physics D: Applied Physics 46 (2) (2013) 024002. doi : 10.1088/0022-3727/46/2/024002

URL http://stacks.iop.org/0022-3727/46/i=2/a=024002?key=crossref. 4eafd3f1793eb077f06cb3fce369da86

[6] Z. S. Bittner, S. Hellstroem, S. J. Polly, R. B. Laghumavarapu, B. Liang, D. L. Huffaker, S. M. Hubbard, Investigation of optical transitions in InAs/GaAs(Sb)/AlAsSb quantum dots using modulation spectroscopy, Applied Physics Letters 105 (25) (2014) 253903. doi : 10. 1063/1 .4904076. URL http://scitation.aip.org/content/aip/journal/apl/105/25/10.1063/1.4904076

[7] A. Mart, N. Lpez, E. Antoln, E. Cnovas, A. Luque, C. R. Stanley, C. D. Farmer, P. Daz, Emitter degradation in quantum dot intermediate band solar cells, Applied Physics Letters 90 (23) (2007) 233510. doi: 10.1063/1.2747195. URL http://scitation.aip.org/content/aip/journal/apl/90/23/10.1063/1.2747195

[8] M. Grundmann, O. Stier, D. Bimberg, InAs/GaAs pyramidal quantum dots: Strain distribution, optical phonons, and electronic structure, Physical Review B 52 (16) (1995) 11969-11981. doi : 10.1103/ PhysRevB . 52 . 11969

URL http://link.aps.org/doi/10.1103/PhysRevB.52.11969

[9] S. M. Hubbard, C. D. Cress, C. G. Bailey, R. P. Raffaelle, S. G. Bailey, D. M. Wilt, Effect of strain compensation on quantum dot enhanced GaAs solar cells, Applied Physics Letters 92 (12) (2008) 123512. doi : 10.1063/1.2903699.

URL http://link.aip.org/link/APPLAB/v92/i12/p123512/s1\&Agg=doi 
[10] V. Popescu, G. Bester, M. C. Hanna, A. G. Norman, A. Zunger, Theoretical and experimental examination of the intermediate-band concept for strain-balanced (In,Ga)As/Ga(As,P) quantum dot solar cells, Physical Review B 78 (20) (2008) 205321. doi : 10.1103/PhysRevB.78.205321. URL http://link.aps.org/doi/10.1103/PhysRevB.78.205321

[11] T. Sugaya, R. Oshima, K. Matsubara, S. Niki, InGaAs quantum dot superlattice with vertically coupled states in InGaP matrix Journal of Applied Physics 114 (1) (2013) 014303. doi : 10 . 1063/1.4812567. URL http://scitation.aip.org/content/aip/journal/jap/114/1/10.1063/1.4812567

[12] N. J. Ekins-Daukes, K. Kawaguchi, J. Zhang, Strain-Balanced Criteria for Multiple Quantum Well Structures and Its Signature in X-ray Rocking Curves, Crystal Growth \& Design 2 (4) (2002) 287-292. doi: $10.1021 / \mathrm{cg} 025502 \mathrm{y}$.

URL http://dx.doi.org/10.1021/cg025502y

[13] C. G. Bailey, S. M. Hubbard, D. V. Forbes, R. P. Raffaelle, Evaluation of strain balancing layer thickness for InAs/GaAs quantum dot arrays using high resolution x-ray diffraction and photoluminescence, Applied Physics Letters 95 (20) (2009) 203110. doi:10.1063/1.3264967

[14] A. Freundlich, M. Gunasekera, C. Rajapaksha, I. Rusakova, Epitaxy of high aspect ratio and wettinglayer-free InAs quantum dots on (Al)GaAs, in: 2012 38th IEEE Photovoltaic Specialists Conference (PVSC), 2012, pp. 001895-001897. doi : 10.1109/PVSC.2012.6317963

[15] I. Vurgaftman, J. R. Meyer, L. R. Ram-Mohan, Band parameters for IIIV compound semiconductors and their alloys Journal of Applied Physics 89 (11) (2001) 5815-5875. doi: doi:10.1063/1. 1368156 .

URL http://jap.aip.org/resource/1/japiau/v89/i11/p5815_s1

[16] L. Vegard, Die Konstitution der Mischkristalle und die Raumfllung der Atome, Zeitschrift fr Physik 5 (1) (1921) 17-26. doi : 10 . 1007/BF01349680

URL http://link. springer.com/article/10.1007/BF01349680

[17] S. Adachi, Properties of group-IV, III-V and II-VI semiconductors, John Wiley \& Sons, Chichester, England; Hoboken, NJ, 2005.

[18] S. Polly, D. Forbes, K. Driscoll, S. Hellstrom, S. Hubbard, Delta-Doping Effects on Quantum-Dot Solar Cells, IEEE Journal of Photovoltaics 4 (4) (2014) 1079-1085. doi:10.1109/JPHOTOV.2014. 2316677.

[19] J. W. Matthews, A. E. Blakeslee, Defects in epitaxial multilayers: I. Misfit dislocations, Journal of Crystal Growth 27 (1974) 118-125. doi:10.1016/S0022-0248(74)80055-2.

URL http://www.sciencedirect.com/science/article/pii/S0022024874800552

[20] E. A. Fitzgerald, Dislocations in strained-layer epitaxy: theory, experiment, and applications Materials Science Reports 7 (3) (1991) 87-142. doi : 10.1016/0920-2307(91)90006-9 URL http://www.sciencedirect.com/science/article/pii/0920230791900069

[21] J. E. Ayers, Heteroepitaxy of Semiconductors: Theory, Growth, and Characterization, CRC Press, 2007.

[22] S. Adachi, P. Capper, S. Kasap, A. Willoughby, Properties of Semiconductor Alloys: Group-IV, III-V and II-VI Semiconductors, 1st Edition, Wiley, Chichester, U.K, 2009. 
[23] S. Polly, A. J. Grede, III-V strain compensation calculator (Feb 2016). doi:doi:10.4231/ D3KP7TS25.

URL https://nanohub.org/resources/21919 


\section{List of Figures}

1 Diagram of superlattice repeat unit (SL) composed of WL + QD or QW and SC, for a) CET for two layers from Section 2.1 b) CET for $n$ layers from Section 2.1| c) MCET Cylinder

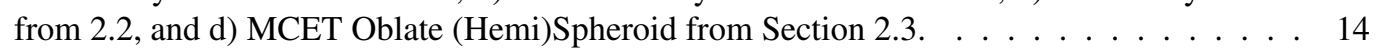

2 Experimental elastic stiffness constants $C_{11}$ from [15] with fits by Adachi [17] and this work using values in Table 1 and Equation 18 . . . . . . . . . . . . . . . . . 15

3 Experimental elastic stiffness constants $C_{12}$ from [15] with fits by Adachi [17] and this work using values in Table 1 and Equation $18 \ldots \ldots \ldots \ldots \ldots \ldots$

$4 \quad$ Required $\mathrm{GaAs}_{(y)} \mathrm{P}_{(1-y)}$ strain compensation layer thickness for InAs QDs on a GaAs substrate as calculated by equations used in Section 2.1 (CET), Section 2.2 (MCET Cyl.), and Section 2.3 (MCET Obl. Sph.), using either literature/interpolated (i), or empirically calculated (e) material parameters. . . . . . . . . . . . . . . . . . . . . . 17

5 Delta thickness (experimentally grown SC thickness minus modeled required SC thickness) of the SC layer versus the delta of the $0^{\text {th }}$ order SL peak (experimentally determined $0^{\text {th }}$ order peak location relative to the Bragg peak minus dynamical scattering calculated $0^{\text {th }}$ order SL peak) for interpolated (i) and empirically determined (e) material parameters. Lines are extended linear fits to each dataset. . . . . . . . . . . . . . . . . . . . . . . . 18

$6 \quad$ Critical/maximum number of SL repeat units using a $\operatorname{GaAs}_{(y)} \mathrm{P}_{(1-y)}$ strain compensation layer, and both InAs QD only and InAs WL only, on a GaAs substrate as calculated by equations used in Section 3.4 and Section 2.1 (CET), Section 2.2 (Cyl.), and Section 2.3 (Obl. Sph.), using either literature/interpolated (i) material parameters. . . . . . . . . . . . . 19 

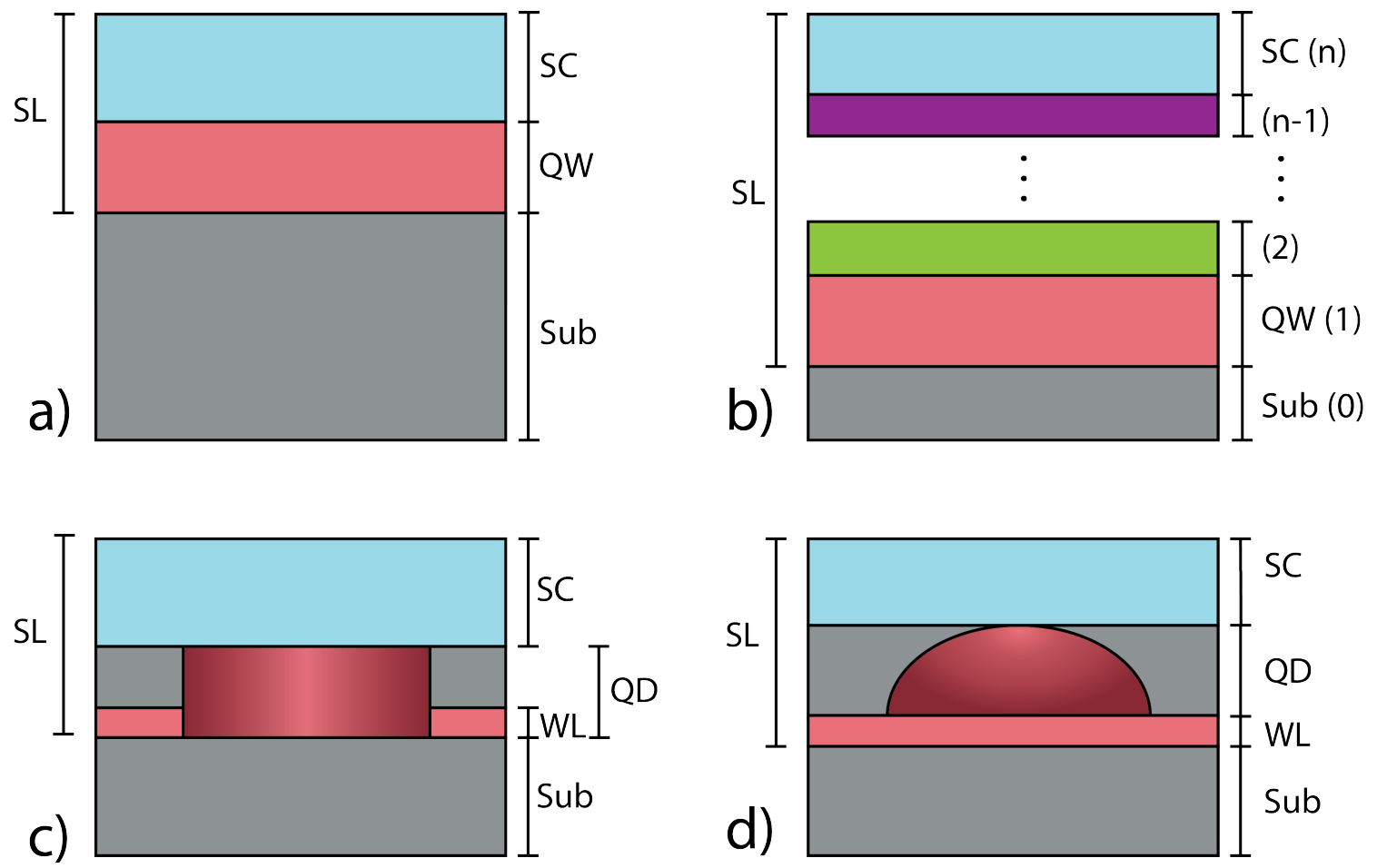

Figure 1: Diagram of superlattice repeat unit (SL) composed of WL + QD or QW and SC, for a) CET for two layers from Section 2.1 b) CET for $n$ layers from Section 2.1 c) MCET Cylinder from 2.2 and d) MCET Oblate (Hemi)Spheroid from Section 2.3 


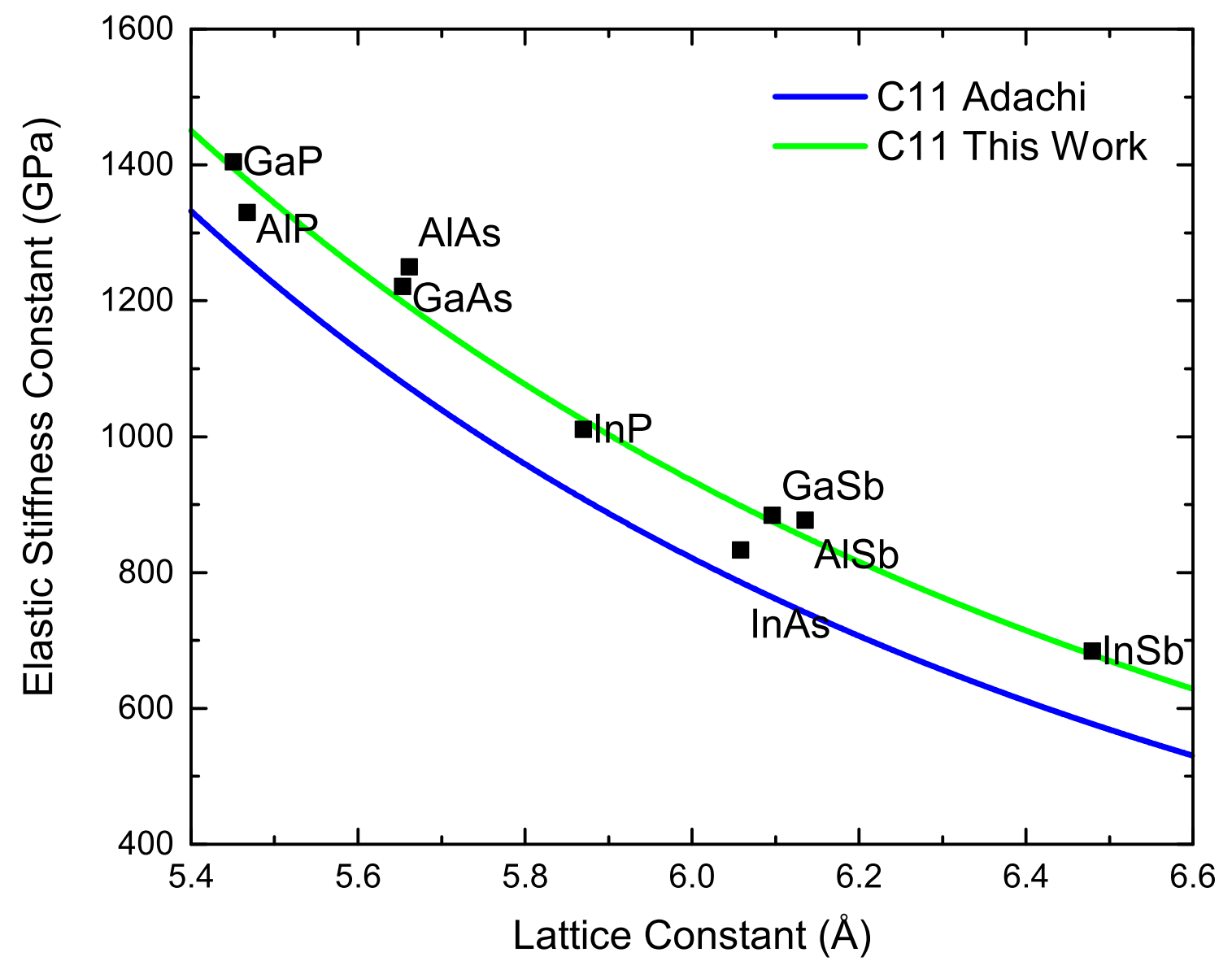

Figure 2: Experimental elastic stiffness constants $C_{11}$ from [15] with fits by Adachi [17] and this work using values in Table 1 and Equation 18 


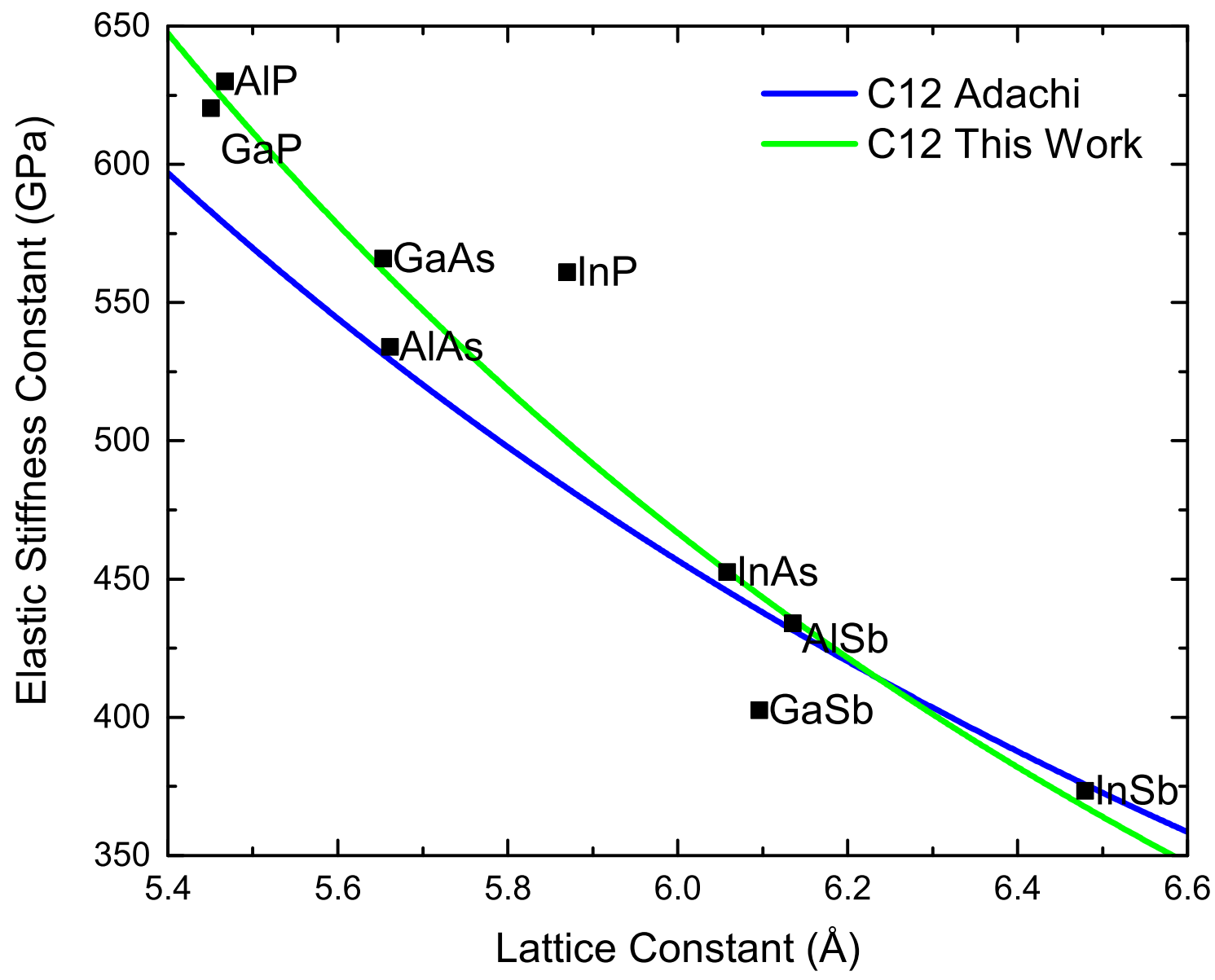

Figure 3: Experimental elastic stiffness constants $C_{12}$ from [15] with fits by Adachi [17] and this work using values in Table 1 and Equation 18 


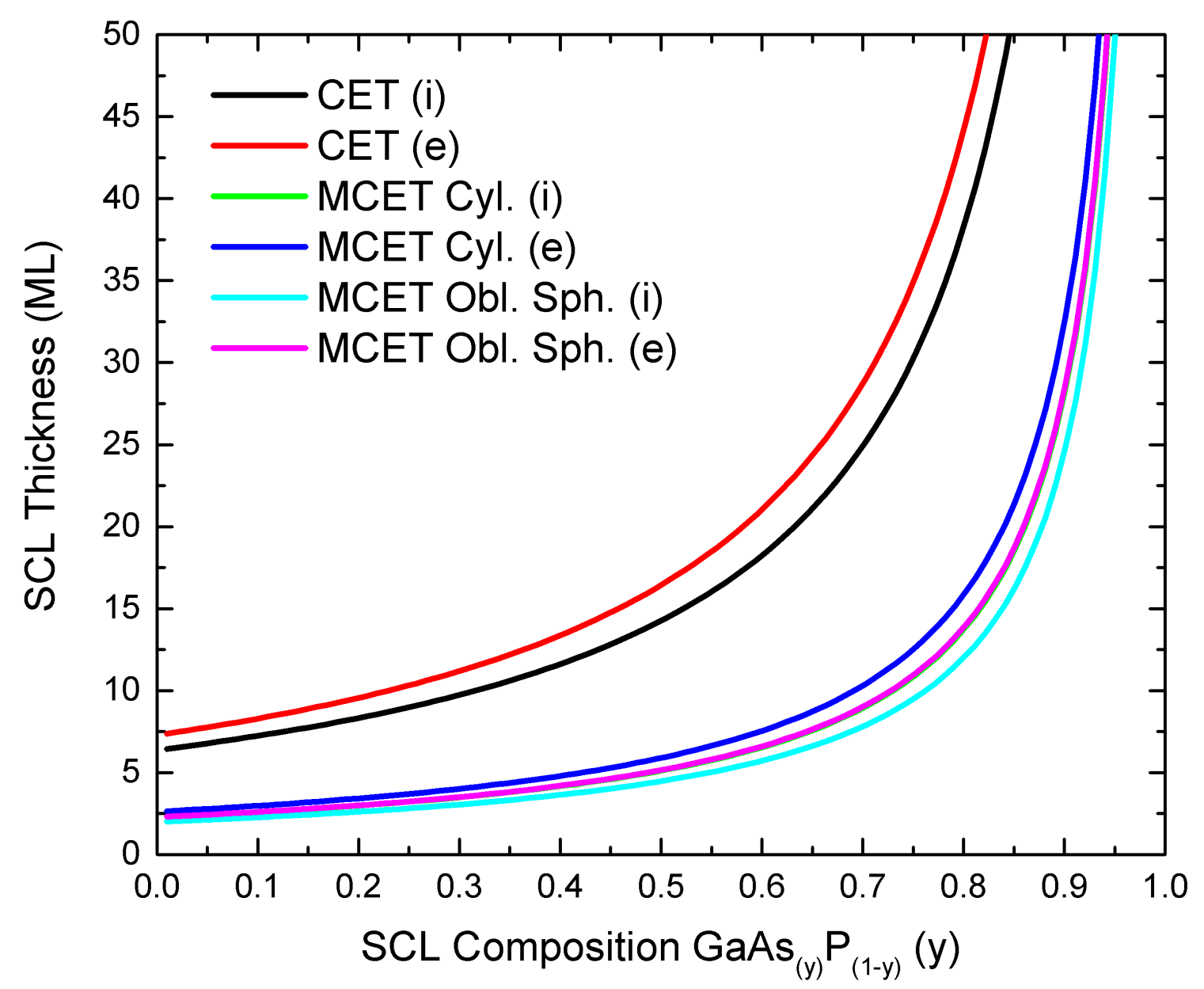

Figure 4: Required $\operatorname{GaAs}_{(y)} \mathrm{P}_{(1-y)}$ strain compensation layer thickness for InAs QDs on a GaAs substrate as calculated by equations used in Section 2.1.(CET), Section 2.2 (MCET Cyl.), and Section 2.3 (MCET Obl. Sph.), using either literature/interpolated (i), or empirically calculated (e) material parameters. 


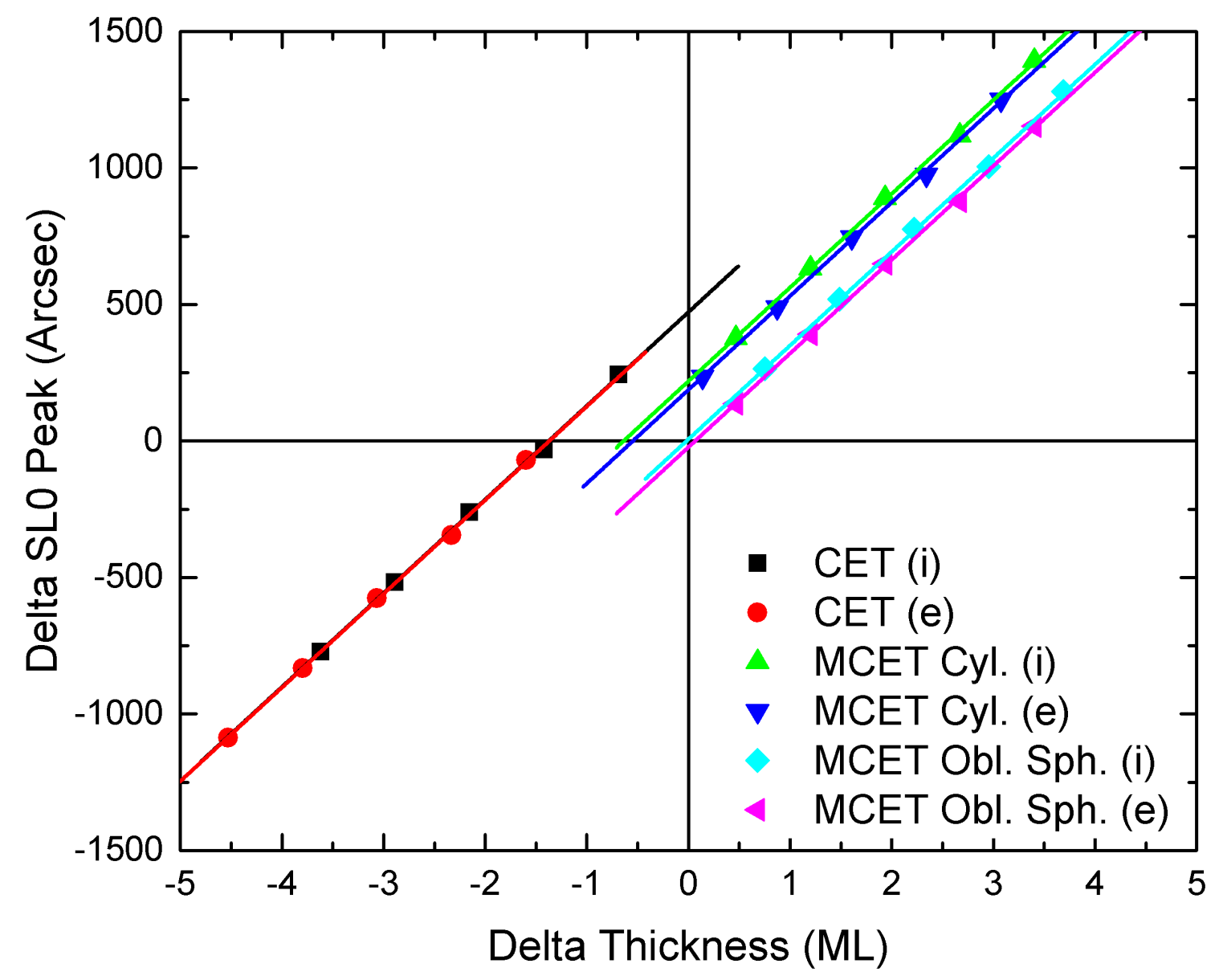

Figure 5: Delta thickness (experimentally grown SC thickness minus modeled required SC thickness) of the SC layer versus the delta of the $0^{\text {th }}$ order SL peak (experimentally determined $0^{\text {th }}$ order peak location relative to the Bragg peak minus dynamical scattering calculated $0^{\text {th }}$ order SL peak) for interpolated (i) and empirically determined (e) material parameters. Lines are extended linear fits to each dataset. 


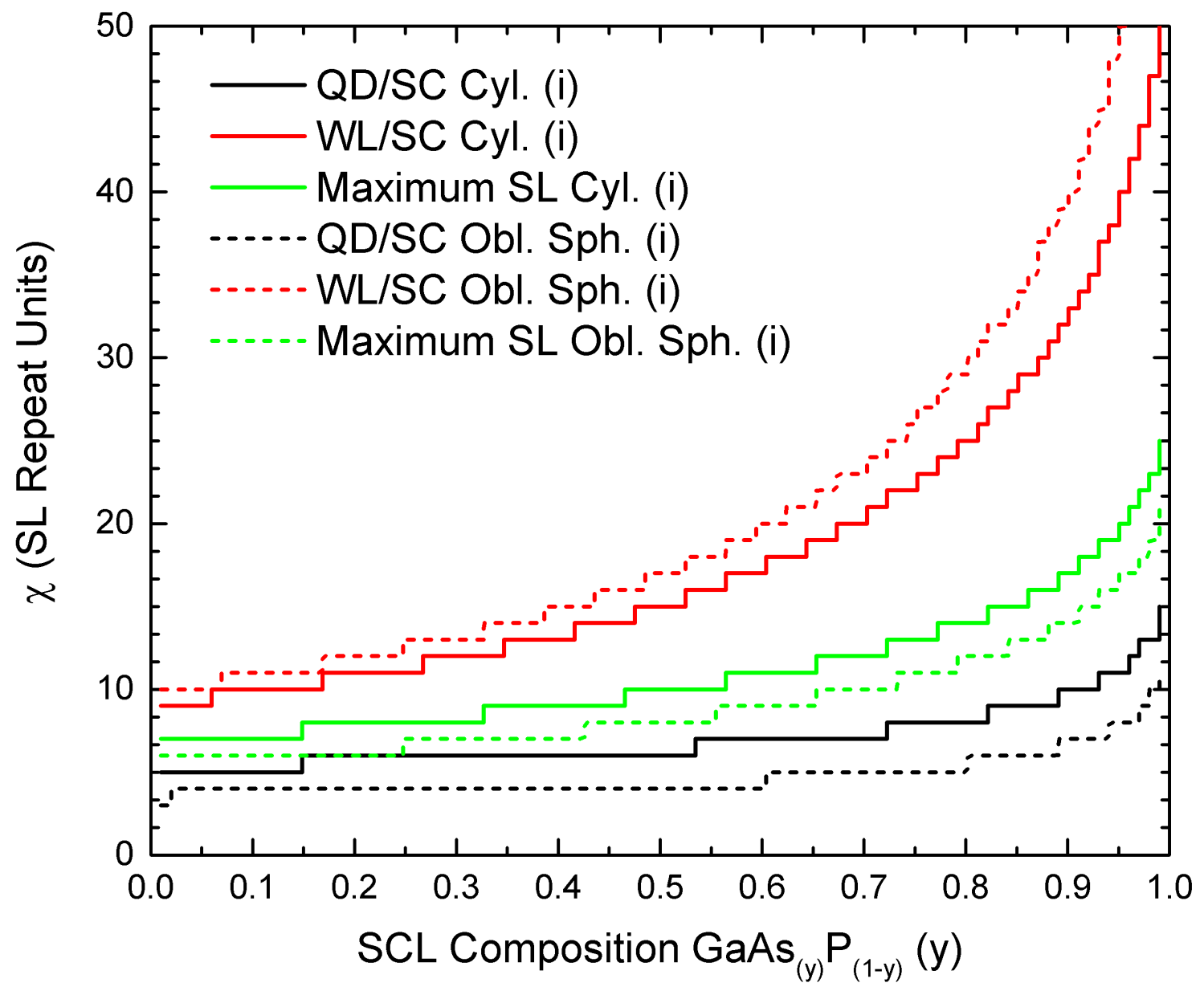

Figure 6: Critical/maximum number of SL repeat units using a $\operatorname{GaAs}_{(y)} \mathrm{P}_{(1-y)}$ strain compensation layer, and both InAs QD only and InAs WL only, on a GaAs substrate as calculated by equations used in Section 3.4 and Section 2.1.(CET), Section 2.2. (Cyl.), and Section 2.3 (Obl. Sph.), using either literature/interpolated (i) material parameters. 


\section{List of Tables}

$1 \quad$ Equation $|18|$ Coefficients for Empirical Fit to Stiffness Constants. . . . . . . . . . . . . . . 21 
Table 1: Equation 18 Coefficients for Empirical Fit to Stiffness Constants.

\begin{tabular}{ccc}
\hline$C_{j k}$ & $\alpha$ & $\beta$ \\
\hline$C_{11}$ & -4.166 & 9.701 \\
$C_{12}$ & -3.105 & 7.104 \\
\hline
\end{tabular}

